\title{
Does rituximab improve clinical outcomes of patients with thyroid-associated ophthalmopathy? A systematic review and meta-analysis
}

\author{
Changjun Wang, Qingyao Ning, Kai Jin, Jiajun Xie and Juan Ye
}

\begin{abstract}
Background: The current therapies of thyroid-associated ophthalmopathy (TAO) were still a challenging matter. In this study, we aimed to contrast the impact of before- after rituximab (RTX) therapy in the patients with TAO.

Methods: We searched the PubMed, EMBASE, and SCOPUS databases for articles published up to July 3, 2017. Fixed- or random-effects meta-analysis was used to provide pooled estimates of standard mean difference (SMD) both the primary outcome from clinical activity score (CAS), and secondary outcomes from thyrotropin receptor antibody (TRAb), proptosis, thyroid stimulating hormone (TSH), and interleukin-6 (IL-6) levels. In addition, the quality and each study was assessed using either the Newcastle Ottawa Scale (NOS) or the Cochrane Risk of Bias tool, and reliability of the meta-analytic result using the Grading of Recommendations Assessment, Development and Evaluation (GRADE).

Results: Of the 839 articles initially searched, 11 studies were finally eligible for inclusion. Subgroup analysis results showed that comparing with initial value, there was a decline in CAS at 1,3,6,12 month after RTX treatment, decreased TRAbs level at 6,12 month, proptosis improvement at least 1 month, unchanged IL-6 level at 6 month, decreased TSH level at 3 month but unchanged at 12 month. All included studies were classified as good quality.

Conclusions: The pooled data suggested that the preliminary effects of RTX treatment on TAO might be promising. However, more large-sample and high-quality studies targeting RTX use during this disease and longterm surveillance of prognosis are urgently needed.
\end{abstract}

Keywords: Inflammation, Orbit, Drugs, Eye lids, Vision

\section{Background}

Thyroid-associated ophthalmopathy (TAO) is an autoimmune inflammatory orbital disease, which usually is caused by $20-50 \%$ of patients with Graves' disease (GD) [1] and has the symptom of bilateral or unilateral eyeball protrusion, eyelid swelling, edema of periorbital tissue, and upper or lower eyelid retraction [2]. It was reported that lymphocytes B played an important role in TAO [3]. $B$ cells could induce immune function via antigen presentation, co-stimulatory molecules expression and antibodies production. They can also be differentiated into

\footnotetext{
* Correspondence: yejuan@zju.edu.cn

Department of Ophthalmology, the Second Affiliated Hospital of Zhejiang

University, College of Medicine, Hangzhou 310009, China
}

antibody-producing plasma cells, which not only caused host defense, but also identified their own tissues, resulting in autoimmunity [4]. In addition, B cells produced cytokines that induced fbroblasts to generate glycosaminoglycan causing fluid and periorbital edema. In recent years, there have been many treatments for TAO, but adverse effects in follow-up should be cautious, such as hypertension, diabetes, stress ulcer, and osteoporosis during immunosuppressive therapies [5], retinopathy, neuropathy, and cataracts in single radiation therapy or combining with oral or intravenous steroids [6], around $20 \%$ to $25 \%$ of nonresponders after intravenous pulses of corticosteroids [7]. 
Rituximab (RTX) is a chimeric mouse monoclonal antihuman CD20 antibody against B-cell proliferation and maturation. RTX has been allowed since 2006 for treating rheumatoid arthritis and had a chance to become a candidate for the treatment of some other autoimmune diseases [8]. In recent years, although some case series have shown that RTX treatment in severe TAO may benefit patients, its application was restricted to case reports and uncontrolled studies, which was a lack of large-scale prospective studies and the effectiveness was still inconsistent. Therefore, we did a systematic review and aimed to check whether the CAS activity improved 1 month or more after RTX treatment for persons with TAO.

\section{Methods}

\section{Search strategy}

We conducted the systematic review according to the Preferred Reporting Items for Systematic Reviews and Meta-Analyses (PRISMA) statement [9]. To assess the evidence on this issue, a broad literature search was independently initiated by Changjun Wang and Qingyao Ning through the PubMed, EMBASE, and SCOPUS databases for articles published up to July 3, 2017 (Appendix 1). Search terms included the keyword terms using rituximab, Graves' ophthalmopathy, thyroid associated orbitopathy, thyroid eye disease. Discrepancy was finally resolved by the senior author (Juan Ye). All literatures were retrieved with no language restrictions. If detailed data were available from online reports, we applied for it directly from authors. These literatures were managed and duplicates were removed through Endnote X7 software (Changjun Wang and Qingyao Ning).

\section{Study selection}

The criteria deciding whether an article was included were as following: (1) cohort study or randomized controlled trial including RTX treatment for patients with TAO; (2) clinical data involving before-after RTX treatment would be available; (3) original articles including one or more parameters of CAS, TRAbs, proptosis, TSH, and IL-6 levels after clinical follow-up of at least 1 month. Studies would be removed if they were case reports, reviews, letters, or conference abstracts without full text.

\section{Data extraction and quality assessment}

Kai Jin and Jiajun Xie extracted these data from each eligible article: authors' names, the type of study, study period, country, age, gender, smoking status, followup. In this analysis, disease activity was estimated according to the seven-point CAS using Snellen chart based on the classical signs of inflammation (ocular pain, eyelid redness, eyelid swelling and fading eyesight) [10]. For each of included studies, CAS was calculated to examine the clinical improvement of these patients at the each ophthalmological visit. Instruments such as the Cochrane risk of bias tool for trials and the Newcastle-Ottawa Scale (NOS) for observational studies were also crosschecked by Changjun Wang and Kai Jin. Quality was assessed using the NOS and Cochrane and reliability of results using GRADE by Changjun Wang and Kai Jin [11, 12]. This was done in duplicate with disagreements handled by discussion with a final arbiter (Juan Ye).

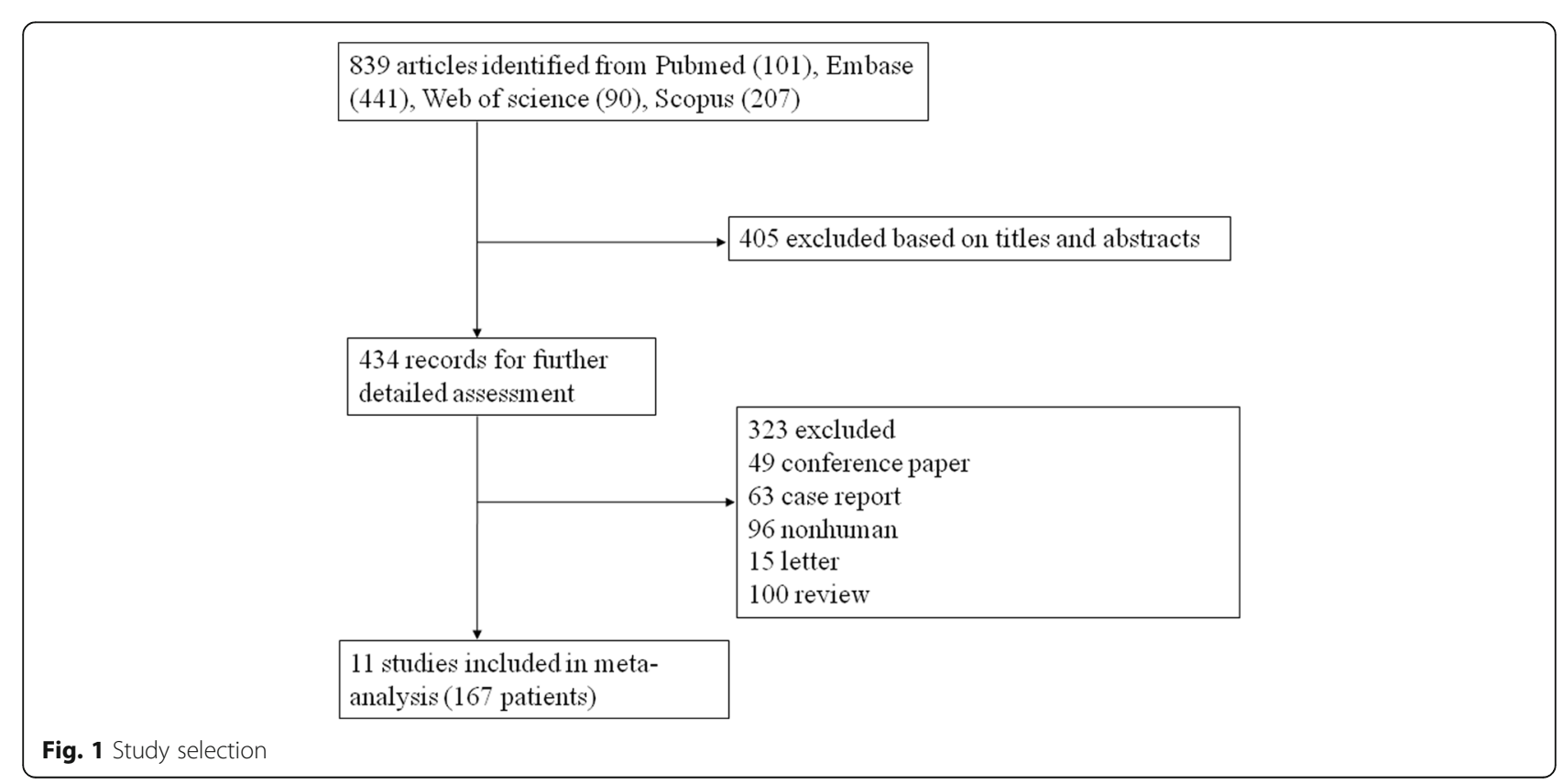




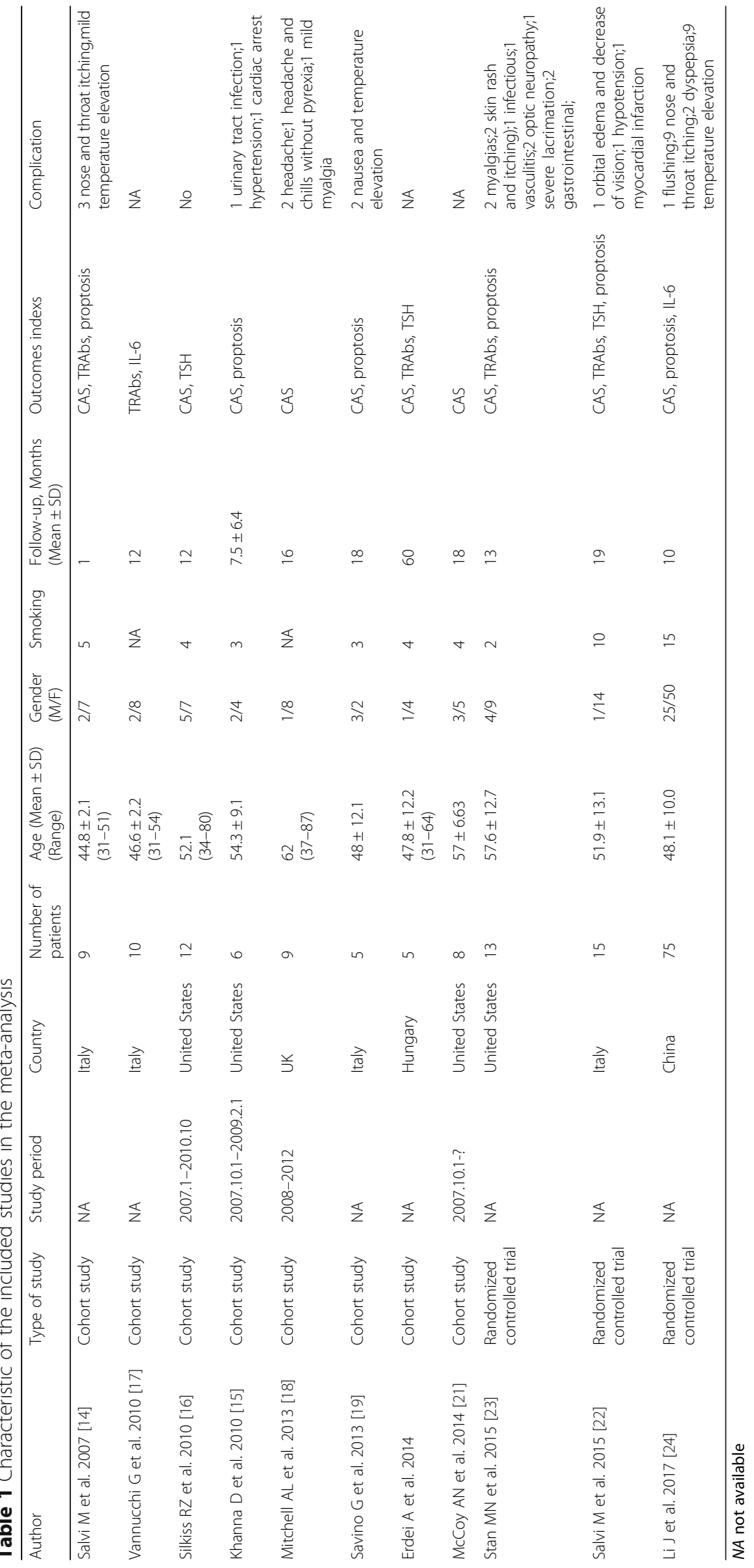




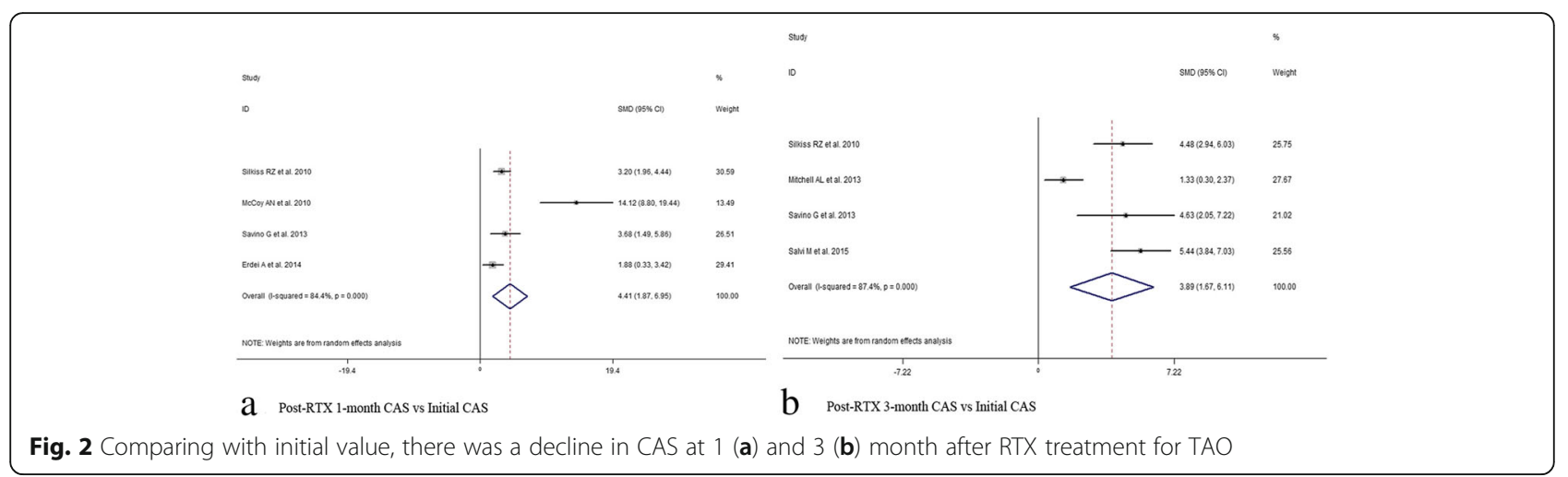

\section{Statistical analysis}

Based on the initial baseline value, the meta-analysis compared the outcome changes at different time interval (1,3,6,12 month, respectively) after RTX therapy, in view of mean CAS as the primary outcome, and mean of TRAbs, proptosis, TSH, IL-6 levels as secondary outcomes. Data were pooled and calculated their SMDs and the $95 \%$ confidence interval (CI) for each subgroup when at least two studies focused on the outcome (Additional file 1: Supplementary files). The metaanalysis was conducted by the Mantel-Haenszel method through Stata 12.0 software [13]. Statistical heterogeneity was estimated using the chi-square test $\left(X^{2}\right), \tau^{2}$ and $I^{2}$ statistic. Where $p<0.05$ or $I^{2}>50 \%$ appeared indicating moderate to large statistical heterogeneity, then a random-effects model was applied. We performed subgroup analysis for heterogeneity according to CAS, proptosis, TRAbs, TSH and IL-6. Otherwise, a fixed-effect model was performed. In addition, the impact of study quality on the results was evaluated by sensitivity analysis, and Egger's tests were assessed for potential publication bias.

\section{Results}

\section{Characteristics of eligible studies in the final analysis}

Our initial search found 839 citations, and after review of title and abstract, 323 studies were recruited for further full-text reading, of which 11 articles were finally available for inclusion [14-24]. The selection flow was showed in Fig. 1. In this study, the mean age of patients was from 44.8 to 62.0 years old. A total of the population consisted of 49 (29.3\%) men and 118 (70.7\%) women, and almost one third smoked. Nearly one half of the participants were from China (44.9\%), followed by Europe (31.7\%) and North America (23.4\%). The mean follow-up of these studies were from 1 to 60 months. Regarding adverse events, mild temperature elevation, nose and throat itching, headache, myalgias, optic neuropathy, orbital edema and decrease of vision, dyspepsia were reported (Table 1). Methodological quality in included studies were acceptable by Newcastle-Ottawa Scale and Cochrane risk of bias tool, in which main drawbacks were the application of few blinding and random distribution, and insufficient sample size, which were reliable appraised through GRADE. Then we tried to minimize it by subgroup analysis based on cohort study and randomized controlled trial, which indicated that most of these results were stable (Additional file 2: Table S1, Additional file 3: Table S2, Additional file 4: Figure S1, Additional file 5: Figure S2).

\section{Heterogeneity test result and subgroup analysis}

In view of the initial CAS value, results indicated significant decrease at 1,3,6,12 months after subsequent RTX treatment (1 month, SMD 95\% CI: 4.77 (2.77-6.77); 3 month,

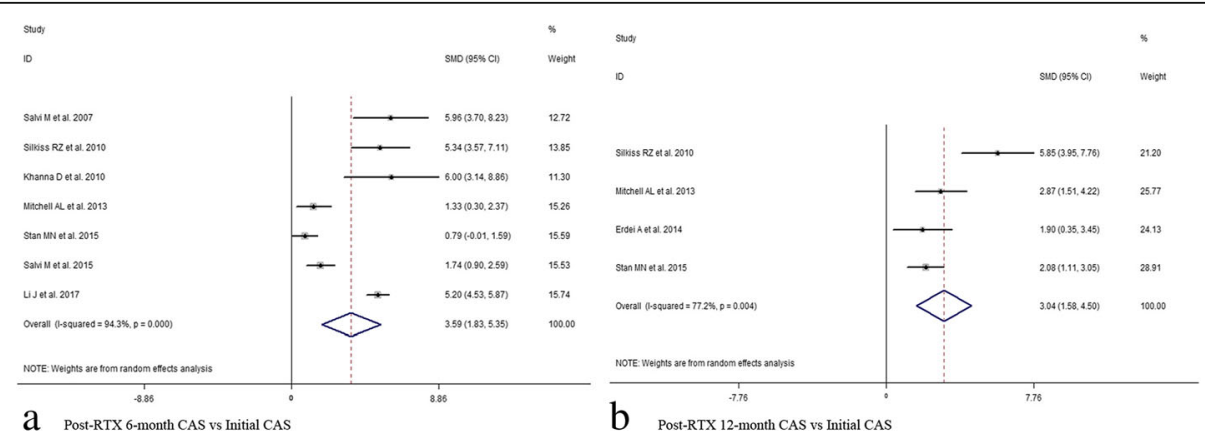

Fig. 3 Comparing with initial value, there was a decline in CAS at 6 (a) and 12 (b) month after RTX treatment for TAO 


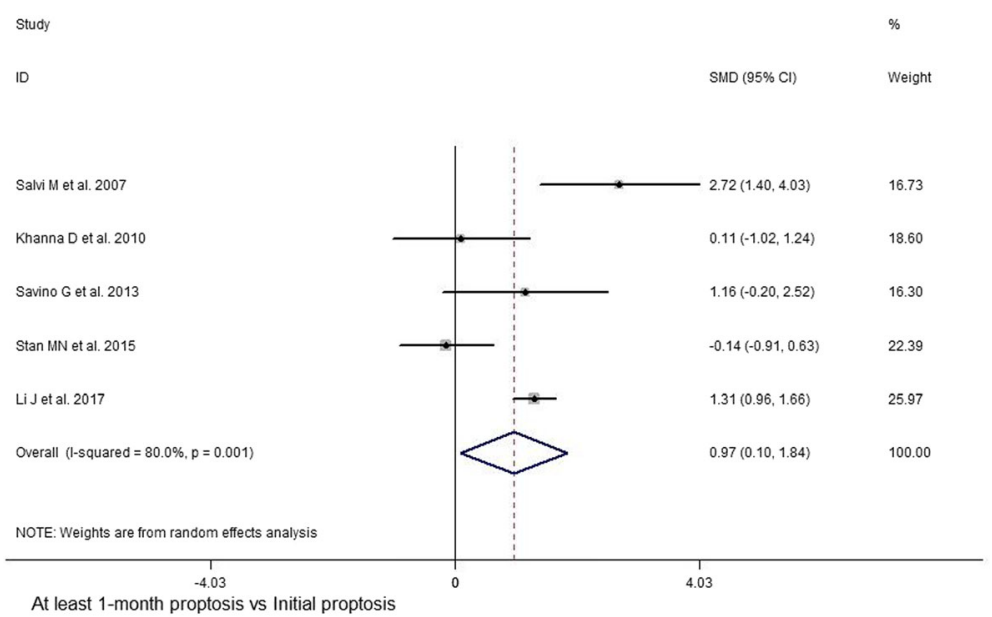

Fig. 4 Comparing with initial value, there was a decrease in proptosis at least 1 month after RTX treatment for TAO

SMD 95\% CI: 3.89 (1.67-6.11); 6 month, SMD 95\% CI: 3.59 (1.83-5.35); 12 month, SMD 95\% CI: 3.04 (1.58-4.50))

(Figs. 2 and 3, Additional file 6: Table S3). At last month of follow-up, proptosis improvement was shown (last month of follow-up, SMD 95\% CI: 0.97 (0.10-1.84)) (Fig. 4). Similarly, pooled data reported TRAbs level were both declining at 6 and 12 months (6 month, SMD 95\% CI: 0.82 (0.401.25); 12 month, SMD 95\% CI: 1.52 (0.80-2.24)) (Fig. 5). TSH level was decreased at 2 month while unchanged at 12 month (2 month, SMD 95\% CI: 0.69 (0.14-1.25); 12 month, SMD 95\% CI: 0.39 (-0.74-1.52)) (Fig. 6). In addition, IL-6 level was also not see an obvious variation (6 month, SMD 95\% CI: 6.47 (-3.26-16.21)) (Fig. 7). In Additional file 6: Table S3, subgroup analysis listed heterogeneity test using $I^{2}$, in which the considerable heterogeneity $\left(I^{2}>75 \%\right)$ was dealed with random-effects model (CAS: 1,3,6,12 month: 84.4\%, 87.4\%, 94.3\%,77.2\%; proptosis: at least 1 month: 80\%; TSH: 12 month: 76.7\%; IL-6: 6 month: 99.3\%).

\section{Sensitivity analysis and publication bias}

We checked the effect of each of these studies through sensitivity analyses, and all were in accordance with our main results. Egger's test suggested no publication bias was in this study (Additional file 6: Table S3).

\section{Discussion}

Thyroid-associated ophthalmopathy was the main extrathyroidal manifestation of Graves' disease. Treatment was depended on the evaluation of the activity and severity of TAO and focused on the patient's living quality. In this systematic review and meta-analysis, we showed that RTX treatment may confer a favorable improvement against TAO. The improvement of TAO remained stable during the follow-up.

Regarding CAS, which may indicate whether these patients benefit or not from RTX therapy. Pain, redness, swelling and impaired function improved in most patients at 1 month after RTX treatment, and sustained during the 12-month follow-up. As shown in a previous study, there were evident reductions from the mean baseline CAS score 5.5 to finally approximate 1.0 for weeks $4,8,16,24$, 36 , and 52 [16]. A high CAS could help select targeting patients who will benefit from RTX treatment. The eyelids became swollen and visual acuity decreased. The serious signs and symptoms in the baseline may result from the

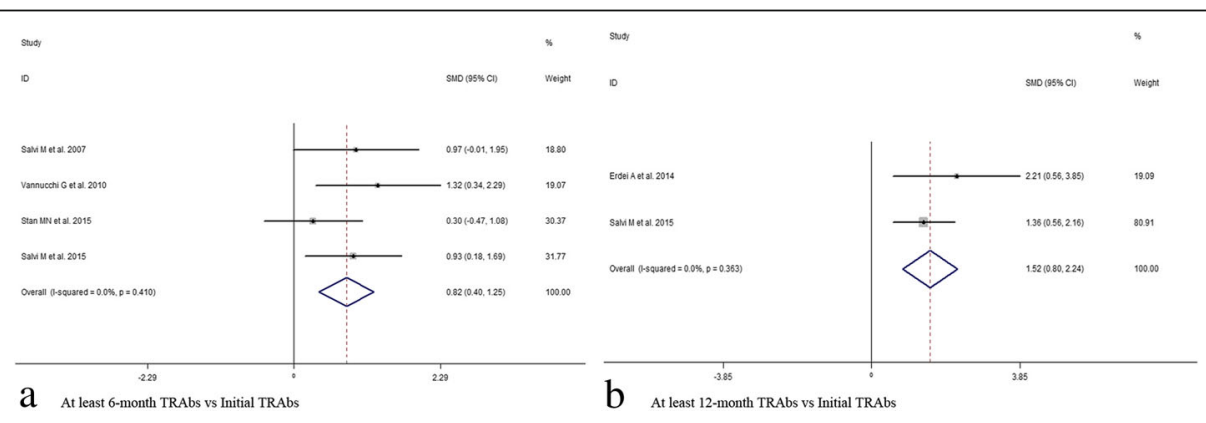

Fig. 5 Comparing with initial value, there was a reduction in TRAbs at 6 (a) and 12 (b) month after RTX treatment for TAO 


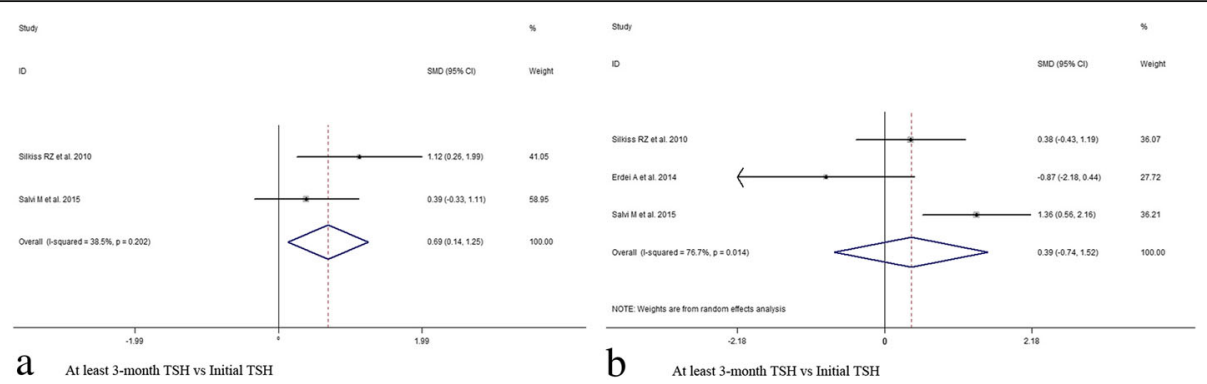

Fig. 6 Comparing with initial value, there was a decline in TSH at 3 month (a) but unchangeable at 12 (b) month after RTX treatment for TAO

inflammation caused by the autoimmune course. Orbital fibroblasts were also considered to be vital in the pathogenesis [10]. Decreased CAS in the majority of individuals meant favorable therapeutic effects. The long-term reduction in CAS scores may suggest it may depend upon the late effects of the drug in some cases.

Recently, unchangeable proptosis was visible in Khanna et al. study [15]. It should be interpreted seriously because the study was only 6 patients and uncontrolled. But in this study, RTX therapy was effective in ameliorating disease severity, as seen in the significant improvement of proptosis and soft tissue inflammation. Furthermore, previous study found that RTX associated with the consumption and resistance of mature B lymphocytes, contributing to control inflammation [25].

Besides CAS, reduction in TRAbs level was observed at 6month and 12-month observation period after RTX use. However, it was reported that serum TRAbs levels were not changed significantly, and was slightly negatively associated with time during 75-week follow-up [14]. Vannucchi et al. found that serum TRAbs have not reduced obviously in TAO cases before 30 weeks since treatment [17]. Based on the previous limited TAO cases treated by RTX, the pooled changes in current results may because TRAb were associated significantly with TAO clinical activity [26]. During the different disease phase, participants with severe TAO have more serum TRAb levels than those with mild-moderate TAO [27]. Although it was possible to include the involvement of B cells, TRAb and cytokines, the detailed mechanism of decreased TRAb levels was still unknown [28]. In addition, the fluctuation of TSH concentration also existed in short- and long-term observation period.

There was a weakly reduced trend in IL-6, which were around the normal range. However, it was different from the prior studies [29]. IL-6 secreted by diverse cells like T and B lymphocytes, monocytes, and fibroblasts could participate in stimulating $\mathrm{T}$ cell, triggering immunoglobulin secretion, also have impacts in fibroblasts and macrophages in TAO [30, 31]. It was likely that blocking IL-6 might be a promising therapy in TAO. But the inapparent results in current meta-analysis could be owing to the small number of participants. Thus large-scale researches were needed in order to make reliable conclusions.

There were several limitations of our review that should be interpreted. First, we have only limited sample size to hardly explore the potential impact of other risk factors such as age, gender, smoking status, dosage of RTX. Second, the orbital variation after RTX treatment could be susceptible to the previous medication of intravenous or oral corticosteroid. Third, subgroup analysis was on the basis of aggregate data, which could mask diversity within individual level and interaction between factors.

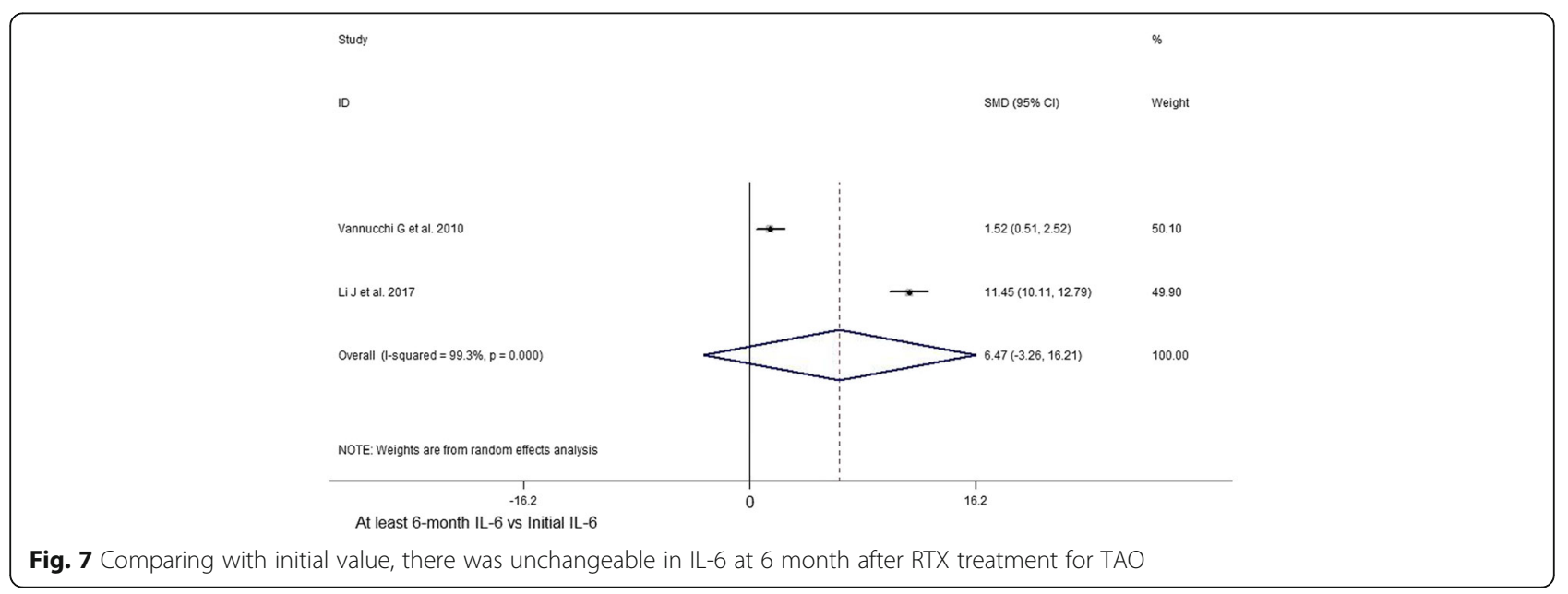


In spite of these, the strengths of this review were that we had a systematic search according to prespecified strategies, and tried to find additional studies. We cross-checked methodological decisions and the influence of each study through sensitivity analyses, and showed the robust results, which were similar to those previous trials, making the results more faithworthy in practice in many countries.

\section{Conclusions}

In short, our analysis of existing empirical evidences suggested that the preliminary effects of RTX treatment on TAO might be promising. We initiated the systematic review of RTX therapy of patients with TAO, which tried to fill in knowledge gaps. However, more large-sample and high-quality studies targeting RTX use during this disease and long-term surveillance of prognosis are urgently warranted.

\section{Appendix}

Search strategy:

Pubmed (1950-present)

1. (rituximab OR RTX)

2. (Graves OR thyroid)

3. (ophthalmopathy OR (eye disease))

4. 1 AND 2 AND 3

5. "rituximab" [Mesh]

6. "Graves ophthalmopathy"[Mesh]

7. 5 AND 6

8. 4 OR 7

Embase(1980-present)

1. 'rituximab':ab,ti

2. 'RTX':ab,ti

3. 1 OR 2

4. 'Graves':ab,ti

5. 'thyroid':ab,ti

6. 4 OR 5

7. 'ophthalmopathy':ab,ti

8. 'eye disease':ab,ti

9. 7 OR 8

10.3 AND 6 AND 9

\section{Scoups}

1. TITLE-ABS-KEY (“rituximab")

2. TITLE-ABS-KEY ("RTX")

3. 1 OR 2

4. TITLE-ABS-KEY (“Graves")

5. TITLE-ABS-KEY ("thyroid")

6. 4 OR 5

7. TITLE-ABS-KEY ("ophthalmopathy")
8. TITLE-ABS-KEY ("eye disease")

9. 7 OR 8

10.3 AND 6 AND 9

\section{Additional files}

Additional file 1: Data collection forms. (DOC $70 \mathrm{~kb}$ )

Additional file 2: Table S1. Quality evaluation of included studies using GRADE criteria. (DOC $70 \mathrm{~kb}$ )

Additional file 3: Table S2. Quality evaluation of included studies using NOS. (DOC $38 \mathrm{~kb}$ )

Additional file 4: Figure S1. Judgements about each risk of bias item presented as percentages across all included studies. (TIFF $26 \mathrm{~kb}$ )

Additional file 5: Figure S2. Judgements about each risk of bias item for each included study. (TIFF $24 \mathrm{~kb}$ )

Additional file 6: Table S3. Mean changes for subgroup during RTX for the treatment of TAO. (DOC $46 \mathrm{~kb}$ )

\section{Abbreviations}

CAS: Clinical activity score; Cl: Confidence interval; GD: Graves' disease; GRADE: Grading of recommendations assessment, development and evaluation; IL-6: Interleukin-6; NOS: Newcastle-Ottawa scale; PRISMA: Preferred reporting items for systematic reviews and meta-analyses; RTX: Rituximab; SMD: Standard mean difference; TAO: Thyroid-associated ophthalmopathy; TRAb: Thyrotropin receptor antibody; TSH: Thyroid stimulating hormone

\section{Acknowledgments}

Not applicable.

Funding

Supported by grants from the Medicine and Health Science Technology Project of Zhejiang Province (2015KYA113), the Scientific Research Foundation of Traditional Chinese Medicine of Zhejiang Province (2015ZB031), the National Natural Science Foundation of China (81670888), National key research and development program (2016YFC1100403). These funding had no role in the study design, data collection and analysis, decision to publish, or preparation of the manuscript.

Availability of data and materials

All data supporting our results has been shared in this study.

\begin{abstract}
Authors' contributions
Study concept and design: JY. Acquisition of data: CW, QN, KJ, JX. Analysis and interpretation of data: CW, QN. Drafting of the manuscript: CW. Critical revision of the manuscript for important intellectual content: CW, JY. Statistical analysis: KJ, JX. Obtained funding: CW. Technical, or material support: KJ, JX. Study supervision: JY. All authors read and approved the final manuscript.
\end{abstract}

Ethics approval and consent to participate

Not applicable.

Consent for publication

Not applicable.

Competing interests

The authors state that there is no competing interests regarding the publication of this paper.

\section{Publisher's Note}

Springer Nature remains neutral with regard to jurisdictional claims in published maps and institutional affiliations. 
Received: 5 August 2017 Accepted: 12 January 2018

Published online: 17 February 2018

\section{References}

1. Bahn RS. Graves' ophthalmopathy. N Engl J Med. 2010;362(8):726-38.

2. Smith TJ. Pathogenesis of graves' orbitopathy: a 2010 update. J Endocrinol Investig. 2010;33(6):414-21.

3. Smith TJ, Tsai CC, Shih MJ, Tsui S, Chen B, Han R, et al. Unique attributes of orbital fibroblasts and global alterations in IGF-1 receptor signaling could explain thyroid-associated Ophthalmopathy. Thyroid Official J Am Thyroid Assoc. 2008;18(9):983.

4. Douglas RS, Naik V, Hwang CJ, Afifiyan NF, Gianoukakis AG, Sand D, et al. B cells from patients with Graves' disease aberrantly express the IGF-1 receptor: implications for disease pathogenesis. J Immunol. 2008;181(8):5768.

5. Bartalena L, Lai A, Compri E, Marcocci C, Tanda ML. Novel immunomodulating agents for graves orbitopathy. Ophthalmic Plast Reconstr Surg. 2008;24(4):251.

6. Bradley EA, Gower EW, Bradley DJ, Meyer DR, Cahill KV, Custer PL, et al. Orbital radiation for graves Ophthalmopathy : a report by the American Academy of ophthalmology. Ophthalmology. 2008;115(2):398-409.

7. Kahaly GJ, Pitz S, Hommel G, Dittmar M. Randomized, single blind trial of intravenous versus oral steroid monotherapy in Graves' orbitopathy. J Clin Endocrinol Metab. 2005;90(9):5234.

8. Anolik JH, Barnard J, Owen T, Zheng B, Kemshetti S, Looney RJ, et al. Delayed memory $B$ cell recovery in peripheral blood and lymphoid tissue in systemic lupus erythematosus after B cell depletion therapy. Arthritis Rheum. 2007:56(9):3044.

9. Moher D, Liberati A, Tetzlaff J, Altman DG, Group TP. Preferred reporting items for systematic reviews and meta-analyses: the PRISMA statement. Revista Esp Nutr Hum Diet. 2009;18(3):889-96.

10. Mourits MP, Prummel MF, Wiersinga WM, Koornneef L. Clinical activity score as a guide in the management of patients with Graves' ophthalmopathy. Clin Endocrinol. 1997:47(1):9-14.

11. Balshem H, Helfand M, Schunemann HJ, Oxman AD, Kunz R, Brozek J, et al. GRADE guidelines: rating the quality of evidence-introduction. 2011.

12. Guyatt G, Oxman AD, Akl EA, Kunz R, Vist G, Brozek J, et al. GRADE guidelines: 1. Introduction-GRADE evidence profiles and summary of findings tables. J Clin Epidemiol. 2012;64(4):383-94.

13. Higgins J, Green SE. Cochrane Handbook for Systematic Reviews of Interventions Version 5.1.0. The Cochrane Collaboration (Eds). NaunynSchmiedebergs Archiv für experimentelle Pathologie und Pharmakologie. 2011;2011(14):S38

14. Salvi M, Vannucchi G, Campi I, Currò N, Dazzi D, Simonetta S, et al. Treatment of Graves' disease and associated ophthalmopathy with the antiCD20 monoclonal antibody rituximab: an open study. Eur J Endocrinol. 2007;156(1):33.

15. Khanna D, Chong KK, Afifiyan NF, Hwang CJ, Lee DK, Garneau HC, et al. Rituximab treatment of patients with severe, corticosteroid-resistant thyroidassociated ophthalmopathy. Ophthalmology. 2010;117(1):133-9.

16. Silkiss RZ, Reier AM, Lauer SA. Rituximab for thyroid eye disease. Ophthalmic Plast Reconstr Surg. 2010;26(5):310-4.

17. Vannucchi G, Campi I, Bonomi M, Covelli D, Dazzi D, Currò N, et al, Rituximab treatment in patients with active Graves' orbitopathy: effects on proinflammatory and humoral immune reactions. Clin Exp Immunol. 2010; 161(3):436-43.

18. Mitchell AL, Gan EH, Morris M, Johnson K, Neoh C, Dickinson AJ, et al. The effect of $\mathrm{B}$ cell depletion therapy on anti-TSH receptor antibodies and clinical outcome in glucocorticoid-refractory Graves' orbitopathy. Clin Endocrinol. 2013;79(3):437-42.

19. Savino G, Balia L, Colucci D, Battendieri R, Gari M, Corsello SM, et al. Intraorbital injection of rituximab: a new approach for active thyroidassociated orbitopathy, a prospective case series. Minerva Endocrinol. 2013; 38(2):173-9.

20. Erdei A, Paragh G, Kovacs P, Karanyi Z, Berenyi E, Galuska L, et al. Rapid response to and long-term effectiveness of anti-CD20 antibody in conventional therapy resistant Graves' orbitopathy: a five-year follow-up study. Autoimmunity. 2014;47(8):548-55

21. Mccoy AN, Kim DS, Gillespie EF, Atkins SJ, Smith TJ, Douglas RS. Rituximab (Rituxan) therapy for severe thyroid-associated ophthalmopathy diminishes IGF-1R(+) T cells. J Clin Endocrinol Metab. 2014;99(7):1294-9.
22. Salvi M, Vannucchi G, Currò N, Campi I, Covelli D, Dazzi D, et al. Efficacy of B-cell targeted therapy with rituximab in patients with active moderate to severe Graves' orbitopathy: a randomized controlled study. J Clin Endocrinol Metab. 2015;100(2):422-31.

23. Stan MN, Garrity JA, Carranza Leon BG, Prabin T, Bradley EA, Bahn RS. Randomized controlled trial of rituximab in patients with Graves' orbitopathy. J Clin Endocrinol Metab. 2015;100(2):432.

24. Li J, Xiao Z, Hu X, Yun L, Xing Z, Zhang S, et al. The efficacy of Rituximab combined with 131 I for ophthalmic outcomes of Graves' Ophthalmopathy patients. Pharmacology. 2017;99(3-4):144.

25. Hasselbalch HC. B-cell depletion with rituximab-a targeted therapy for Graves' disease and autoimmune thyroiditis. Immunol Lett. 2003;88(1):85-6.

26. Gerding MN, Meer JWCVD, Broenink M, Bakker O, Wiersinga WM, Prummel MF. Association of thyrotrophin receptor antibodies with the clinical features of Graves' ophthalmopathy. Clin Endocrinol. 2000:52(3):267-71.

27. Eckstein AK, Plicht M, Lax H, Neuhäuser M, Mann K, Lederbogen S, et al. Thyrotropin receptor autoantibodies are independent risk factors for Graves' ophthalmopathy and help to predict severity and outcome of the disease. J Clin Endocrinol Metab 2006;91(9):3464.

28. Minakaran N, Ezra DG. Rituximab for thyroid-associated ophthalmopathy. Cochrane Database Syst Rev. 2013;5(5):CD009226.

29. Salvi M, Vannucchi G, Beck-Peccoz P. Potential utility of rituximab for Graves' orbitopathy. J Clin Endocrinol Metab. 2013;98(11):4291-9

30. Jyonouchi SC, Valyasevi RW, Harteneck DA, Dutton CM, Bahn RS. Interleukin6 stimulates thyrotropin receptor expression in human orbital preadipocyte fibroblasts from patients with Graves' ophthalmopathy. Thyroid. 2001;11(10): 929-34.

31. Pérez-Moreiras JV, Alvarez-López A, Gómez EC. Treatment of active corticosteroid-resistant graves' orbitopathy. Ophthalmic Plast Reconstr Surg. 2014;30(2):162.

\section{Submit your next manuscript to BioMed Central and we will help you at every step:}

- We accept pre-submission inquiries

- Our selector tool helps you to find the most relevant journal

- We provide round the clock customer support

- Convenient online submission

- Thorough peer review

- Inclusion in PubMed and all major indexing services

- Maximum visibility for your research

Submit your manuscript at www.biomedcentral.com/submit
) Biomed Central 